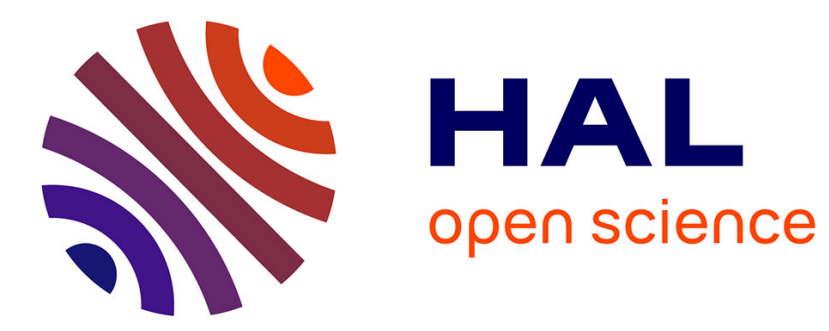

\title{
How to aggregate experts' discount rates: an equilibrium approach
}

\author{
Elyès Jouini, Clotilde Napp
}

\section{To cite this version:}

Elyès Jouini, Clotilde Napp. How to aggregate experts' discount rates: an equilibrium approach. Economic Modelling, 2014, 36, pp.235-243. 10.1016/j.econmod.2013.09.052 . halshs-00927269

\section{HAL Id: halshs-00927269 \\ https://shs.hal.science/halshs-00927269}

Submitted on 12 Jan 2014

HAL is a multi-disciplinary open access archive for the deposit and dissemination of scientific research documents, whether they are published or not. The documents may come from teaching and research institutions in France or abroad, or from public or private research centers.
L'archive ouverte pluridisciplinaire HAL, est destinée au dépôt et à la diffusion de documents scientifiques de niveau recherche, publiés ou non, émanant des établissements d'enseignement et de recherche français ou étrangers, des laboratoires publics ou privés. 


\title{
How to aggregate experts discount rates: an equilibrium approach*
}

\author{
Elyès Jouini ${ }^{\dagger}$ and Clotilde Napp $\$ \S$
}

July 17, 2008

*The financial support of the GIP ANR ("Croyances" project) and of the Risk Foun dation (Groupama chair) is gratefully acknowledged.

†Université Paris Dauphine, Ceremade, F 75016 Paris, France, jouini@cere made.dauphine.fr, phone: + 33144054226

$\ddagger$ CNRS, UMR7088, F 75016 Paris, France

$\S$ Université Paris Dauphine, DRM, F 75016 Paris, France, clotilde.napp@dauphine.fr, phone: + 33144054642 


\title{
How to aggregate experts discount rates: an equilibrium approach
}

\begin{abstract}
We address the problem of a social planner who, as in Weitzman (2001), gathers data on experts' discount rates and wants to infer the socially efficient consumption discount rate. We propose an 'equilibrium approach' and we analyse the expression and the properties of the resulting 'equilibrium discount rate'. We compare our expression for the discount rate with the different expressions that have been previously proposed in the literature. We analyse the impact of shifts in the distributions of experts discount rates. Finally, we apply our approach to Weitzman (2001)'s data to propose discount rates for public sector Cost-Benefit Analysis, in particular for the long term.

Key-words: consumption discount rate; equilibrium discount rate; experts discount rate; hyperbolic discounting; cost-benefit analysis; gamma discounting; divergence of opinion;
\end{abstract}




\section{Introduction}

The appropriate social discount rate to apply in public sector cost-benefit analysis is a contentious issue. This is especially true for long term projects, for which financial markets cannot provide any guideline. As Weitzman (2001, p.261) states "There does not now exist, nor has ever existed, anything remotely resembling a consensus, even -or, perhaps one should say especially- among the 'experts' on this subject".

In this paper we address the problem of a social planner, who gathers data on experts' discount rates and wants to infer the socially efficient discount rate. More precisely, as in Weitzman (2001), the social planner has consulted a group of experts about the discount rate to apply for costs or benefits occurring at a given date $t$, each expert has proposed a discount rate and the problem of the social planner is to aggregate these proposed discount rates into a socially efficient discount rate.

As underlined by e.g. Nordhaus (2007) or Weitzman (2007), there is an important distinction between the utility social discount rate and the consumption social discount rate. The former refers to a pure time preference rate that discounts utility. It reflects the level of impatience or, for long time horizon projects, the relative weights of different people or generations. The latter is the rate used to discount future consumption; it is determined by the time preference rate, but also by the anticipations about the future of the economy. The (extended) Ramsey equation ${ }^{1}$ illustrates $^{2}$ the distinction and the relation between the utility discount rate and the consumption discount rate. Letting $R$ denote the consumption discount rate, and $\rho$ the utility discount rate, Ramsey formula gives the relation $R \quad \rho+\eta \mu \quad \frac{1}{2} \eta(1+\eta) \sigma^{2}$, where $\mu$ is the growth rate of the economy and $\eta$ is the elasticity of marginal utility. Apart in the specific settings of a stationary economy $\left(\begin{array}{lll}\mu & \sigma & 0\end{array}\right)$ or a risk neutral investor $\left(\begin{array}{ll}\eta & 0\end{array}\right)$ or when the wealth and precautionary savings effect cancel out $\left(\mu \frac{1}{2}(1+\eta) \sigma^{2}\right)$, the two rates differ. In this paper, the rates proposed by the experts, as well as the socially efficient discount rate to be inferred, are consumption discount rates, since they are to be applied to cost-benefit analysis. This is also the case in Weitzman (2001); indeed, as Weitzman makes it clear in

\footnotetext{
${ }^{1}$ The original Ramsey equation (Ramsey, 1928) was derived in a deterministic setting $(\sigma=0)$ and is given by $R=\rho+\eta \mu$. The extended Ramsey equation corresponds to a direct generalization in a stochastic setting. For the sake of completeness, we rederive it in the Appendix.
} 
his questionnaire : "What I am here after is the relevant interest rate for discounting real-dollar changes in future goods and services as opposed to the rate of pure time preference on utility" 2 .

Weitzman $(1998,2001)$ deal with this problem by adopting a certainty equivalent approach. In this certainty equivalent approach, the social discount factor is taken to be the (arithmetic) average of the discount factors proposed by the experts. Weitzman (1998) derives properties of the certainty equivalent discount rate and in particular, its convergence towards the lowest individual discount rate, whatever the distribution of experts' discount rates. Weitzman (2001) applies this approach to a specific example. Starting from the results of a survey based on the opinions of 2,160 economists about the consumption discount rate, he estimates the distribution of experts' discount rates and derives an explicit expression for the certainty equivalent discount rate in this case (Gamma discounting). Gollier (2004) underlines that the approach of Weitzman $(1998,2001)$ amounts to ranking the projects according to their expected net present value. By adopting the criterion that projects should be ranked according to their expected future value, Gollier (2004) reaches opposite conclusions and concludes that "both criteria are arbitrary as they do not rely on realistic preferences of human beings towards risk and time" suggesting that an equilibrium analysis is maybe the cost to be paid to make policy recommendations that have an economic sense.

We propose an approach to aggregate experts' discount rates into a consensus discount rate, which relies on an equilibrium analysis. Our approach is the following. We consider that each expert in the panel has consulted an equilibrium model, calibrating it with her own tastes and beliefs parameters, in order to propose her own discount rate. For instance, expert $i$ has applied the Ramsey formula and proposed a discount rate $R^{i}$ that corresponds to her own pure time preference rate $\rho_{i}$ (or as previously underlined, to her own conception of intergenerational equity) and her own beliefs $\left(\mu_{i}, \sigma_{i}\right)$ about the future growth of the economy. The divergence in the proposed individual discount rates $R^{i}$ stems then from divergence in individual tastes and beliefs. We consider that experts' tastes and beliefs are representative in the sense that each of them represents the tastes and beliefs of a portion of the population. It is then natural to adopt as the

\footnotetext{
${ }^{2}$ Moreover, the fact that the given rates are on average equal to $4 \%$ confirms that the experts actually gave their discount rate for consumption.
} 
socially efficient discount rate the equilibrium discount rate in the economy made of agents with the heterogeneous beliefs and tastes of the experts. For example, if the panel is made of three experts, the first expert proposing the discount rate $R^{1}$, the second $R^{2}$ and the third $R^{3}$ corresponding respectively to characteristics $\left(\rho_{1}, \mu_{1}, \sigma_{1}\right),\left(\rho_{2}, \mu_{2}, \sigma_{2}\right)$ and $\left(\rho_{3}, \mu_{3}, \sigma_{3}\right)$, and if we assume that the three experts are equally representative, then we take as the socially efficient discount rate the equilibrium discount rate in an economy made of one third of agents with characteristics $\left(\rho_{1}, \mu_{1}, \sigma_{1}\right)$, one third of agents with characteristics $\left(\rho_{2}, \mu_{2}, \sigma_{2}\right)$ and one third of agents with characteristics $\left(\rho_{3}, \mu_{3}, \sigma_{3}\right)$. This means that we have transformed the problem of aggregating data on heterogeneous discount rates into the problem of aggregating data on heterogeneous beliefs and tastes.

We can then apply the techniques ${ }^{3}$ of Jouini et al. (2008) in order to obtain the expression of the socially efficient discount rate. If we let $A_{t}$ denote the price at date 0 of a zero coupon bond maturing at date $t$, the average discount rate between date 0 and date $t$ is given by $R_{t} \equiv \frac{1}{t} \ln A_{t}$ and the marginal discount rate is given by $r_{t} \equiv \frac{A_{t}^{\prime}}{A_{t}}$. We obtain that both rates are weighted averages of the individual discount rates. They are both decreasing with time and converging to the lowest individual expert discount rate. These properties hold for both constant and decreasing pure time preference rates.

In the present paper we consider logarithmic utility functions. There are essentially two reasons for such a restriction. The first reason is analytical tractability. Indeed, as underlined by Rubinstein (1975), "log utility functions are singular in their capacity to cope with heterogeneous beliefs while not imposing unreasonable restrictions on tastes". This choice enables us to obtain simple formula, while considering reasonable levels of risk aversion ${ }^{4}$. The second reason is the central role of logarithmic utility functions. Jouini et al. (2008) shows that the log-utility setting is central in the analysis of beliefs heterogeneity: some biases are induced when we deal with power utility function $\frac{c^{1-\eta}}{1 \eta}$ with $\eta / 1$, these biases being in opposite directions depending on the position of $\eta$ with respect to 1 . This is then an additional argument in favor of the log-utility setting. Note finally that

\footnotetext{
${ }^{3}$ Jouini et al. (2008) deals with the determination of the equilibrium discount rate in an economy in which agents have heterogeneous beliefs and heterogeneous time preference rates.

${ }^{4}$ The case with more general power utility functions would be much more difficult to handle.
} 
the choice of $\eta \quad 1$ is also made in the Stern Review.

We compare our expression for the socially efficient discount rate with other possible functions of the individual discount rates previously considered in the literature. In particular, our formula are different from Weitzman (1998). The equilibrium discount rate coincides with Weitzman (1998, 2001) certainty equivalent discount rate when all experts have the same pure time preference rate. In a more general setting, the discount rates of the more impatient experts are granted a higher weight. A possible interpretation is as follows. When considering its intertemporal rate of substitution, the group must weigh more the agents with a higher shadow price of the intertemporal budget constraint, i.e. the more impatient members of the group. The overweighting of impatient experts discount rates implies that when tastes and beliefs are independent, the equilibrium discount rate is higher than the certainty equivalent discount rate for all horizons.

We determine the explicit expression of the socially efficient discount rate for specific distributions of the experts' discount rates. We consider Gaussian as well as Gamma distributions. We calibrate the model with a Gamma distribution on Weitzman (2001)'s data. Our results suggest using the following approximation of within-period marginal discount rates for long term public projects: Immediate Future about 5 per cent; Near Future about 4 percent; Medium Future about 3 percent; Distant Future about 1.5 per cent and Far-Distant Future about 0 per cent. Except for the Far-Distant Future, these rates are slightly higher than those obtained by Weitzman (2001) .

Finally, we determine which concepts of stochastic dominance on the distributions of experts/individual discount rates lead to a clear impact on the equilibrium discount rate. We analyze the impact of standard shifts, like first or second stochastic dominance shifts as well as monotone likelihood ratio dominance shifts. The impact of these shifts are different depending on which discount rate (average or marginal) we consider. Roughly speaking, more pessimism, more patience, more doubt as well as more heterogeneity in individual discount rates reduce the equilibrium discount rate.

Note that our approach also permits to aggregate utility discount rates ${ }^{5}$ (pure time discount rates). It suffices to consider the specific case where

\footnotetext{
${ }^{5}$ The problem of aggregating individual utility discount rates has been studied by, among others, Reinschmidt (2002) through a certainty equivalent approach, Nocetti et al. (2008) through a Benthamite approach, Gollier Zeckhauser (2005) through a Pareto optimality approach and Lengwiler (2005) through an equilibrium approach.
} 
there is no beliefs heterogeneity. We obtain that the equilibrium utility discount rate is a weighted average of the individual ones. Our formulas are analogous to those of Lengwiler (2005). They coincide with those of Nocetti et al. (2008) and Gollier and Zeckhauser (2005) only for specific choices of Pareto weights. They differ from those in Reinschmidt (2002), in the same way as our formulas for the consumption discount rate differ from those in Weitzman (1998). We emphasize that, while these papers aim at aggregating individual utility discount rates, the aim of the present paper is to aggregate individual consumption discount rates and to do it through an equilibrium approach.

All proofs are in the Appendix.

\section{Equilibrium discount rate}

Let us consider $n$ experts, who propose different discount rates $\left(R^{i}\right)$ for cost-benefit analysis of public projects as in Weitzman (2001).

We assume that the discount rate proposed by expert $i$ for costs or benefits occurring at date $t$ comes from a general equilibrium model with $\log$ utility and lognormal aggregate consumption at date $t$. According to the extended Ramsey equation, the consumption discount rate $R^{i}$ proposed by expert $i$ is given by

$$
R^{i} \quad \rho_{i}+\mu_{i} \quad \sigma_{i}^{2}
$$

where $\rho_{i}>0, \mu_{i}$ and $\sigma_{i}^{2}$ are respectively the pure time preference rate, the mean and the variance (by unit of time) of the distribution of the growth rate of aggregate consumption that the expert uses in order to calibrate the model. The divergence on the discount rates $\left(R^{i}\right)$ results then from divergence on these parameters.

Assuming that experts differ in their expectation about the growth rate is fairly natural. Indeed, the expected growth rate reflects the opinion about the future. It suffices to look at experts forecasts to realise that there is no consensus about the future of the economy. Indeed, forecasting for the coming year is already a difficult task. It is natural that forecasts for the next century/millennium are subject to potentially enormous divergence. It is doubtful that agents or economists currently have a complete understanding of the determinants of long term economic evolutions. It is also natural to assume that experts differ in their pure time preference rate since it may reflect their point of view about intergenerational equity as well as 
one's level of impatience. The important debate among economists (and also among philosophers) on the notion of intergenerational equity is an illustration of this possible divergence. Some will argue that intergenerational choices should be treated as intertemporal individual choices leading to weigh more present welfare. Others will argue that fundamental ethics require intergenerational neutrality and that the only ethical basis for placing less value on the welfare of future generations is the uncertainty about whether or not the world will exist and whether or not these generations will be present.

The problem now is to determine how to aggregate these experts' recommended discount rates into a consensus discount rate. We consider that the panel experts' tastes and beliefs reflect those of the population. We shall then consider a complete markets economy with heterogeneous agents endowed with the beliefs and tastes chosen by the experts and we shall adopt the equilibrium discount rate in this economy as our consensus discount rate. There is no specific reason to discriminate between the experts, hence we suppose that each expert is representative of the same proportion of the agents in the economy and that all the agents have the same initial endowment.

To summarise, we have

- $N$ groups of agents,

- $w_{i} \equiv$ relative size of group $i$,

- $\rho_{i} \equiv$ pure time preference rate of the agents in group $i$,

- $t \equiv$ the time at which a cost or benefit is incurred, relative to the present time,

- $\ln \mathcal{N}\left(\left(\mu_{i} \frac{1}{2} \sigma_{i}^{2}\right) t, \sigma_{i}^{2} t\right) \equiv$ group $i$ 's anticipated distribution ${ }^{6}$ of aggregate consumption at date $t$,

- $\log$ utility functions,

- $R^{i} \equiv \rho_{i}+\mu_{i} \quad \sigma_{i}^{2}$, the individual discount rate for agents in group $i$, i.e. the equilibrium discount rate that would prevail if the economy was made of group $i$ agents only.

\footnotetext{
${ }^{6}$ This is the case for instance if aggregate consumption is a geometric Brownian motion with drift $\mu_{i}$ and volatility $\sigma_{i}$. We will in Section 4 consider a more general setting with general distributions for aggregate consumption. The formulas are then less easy to handle.
} 
The weights $w_{i}$ model then the distribution of agents'c haracteristics in the economy, which also correspond by construction to the distribution of experts characteristics.

\subsection{Expression of the equilibrium discount rate and properties}

We denote by $A_{t}$ the equilibrium discount factor for horizon $t$, i.e. the price at date 0 of $\$ 1$ at date $t$. We denote by $R_{t} \equiv \frac{1}{t} \ln A_{t}$ the discount rate for horizon $t$, i.e. the rate which if applied constantly for all intervening years would yield the discount factor $A_{t}$. We denote by $r_{t} \equiv \frac{A_{t}^{\prime}}{A_{t}}$ the marginal discount rate for horizon $t$, i.e. the rate of change of the discount factor. We have $R_{t} \quad \frac{1}{t} \int_{0}^{t} r_{s} d s$. Marginal and average rates of discount coincide when the discount rate is constant. In particular, for all $i$, the individual marginal discount rate $r^{i}$ coincides with the individual discount rate $R^{i}$. However, the distinction between the two notions of discount rates can become important when the discount rate is time dependent (Groom et al., 2005).

We easily get, as in Jouini et al. (2008, Proposition 5.1), that the average discount rate $R_{t}$ is given by

$$
R_{t} \equiv \frac{1}{t} \ln \sum_{i}^{N} \frac{w_{i} \rho_{i}}{\sum_{j}^{N}{ }_{1} w_{j} \rho_{j}} \exp \quad R^{i} t
$$

that it decreases with $t$ and that it converges to the lowest proposed rate $R_{\infty} \quad \inf _{i} R^{i}$. Note that our setting is slightly different from the setting in Jouini et al. (2008). Indeed, in Jouini et al. (2008), aggregate consumption follows a specific diffusion process. However, the proofs remain essentially the same. For the sake of completeness, we provide the proof of Equation (1) at the beginning of the Appendix. Moreover, we will show in Section 4 that the formula for $R_{t}$ in Equation (1) remains valid in a very general Arrow Debreu setting, with continuous or discrete time, a finite number or a continuum of agents, and general distributions for aggregate consumption ${ }^{7}$.

Analogously, we easily obtain the following results on the marginal discount rate.

\footnotetext{
${ }^{7}$ In a more general setting, the individual discount rates $R_{t}^{i}$ are not given by the Ramsey formula and may then be dependent upon $t$.
} 
Proposition 1 1. The equilibrium marginal discount rate is given by

$$
r_{t} \equiv \sum_{i=1}^{N} \frac{w_{i} \rho_{i} \exp \left(r^{i} t\right)}{\sum_{j 1}^{N} w_{j} \rho_{j} \exp \left(r^{j} t\right)} r^{i} .
$$

2. In the case of homogeneous beliefs $\left(\mu_{i} \quad \mu, \sigma_{i} \quad \sigma\right)$, the equilibrium marginal discount rate is given by

$$
r_{t} \equiv \sum_{i=1}^{N} \frac{w_{i} \rho_{i} \exp \left(\rho_{i} t\right)}{\sum_{j}^{N}{ }_{1} w_{j} \rho_{j} \exp \left(\rho_{j} t\right)} \rho_{i}+\mu \quad \sigma^{2} .
$$

3. The discount rate $r_{t}$ decrease with $t$, and the asymptotic equilibrium discount rate is given by the lowest individual discount rate, i.e. $r_{\infty}$ $\inf _{i} r^{i} \quad R_{\infty}$.

As in the certainty equivalent approach of Weitzman (1998), the consensus discount factors obtained through our equilibrium approach are averages of the individual discount factors proposed by the experts. However, except in the case of homogeneous pure time preference rates, i.e. $\rho_{i} \quad \rho$ for all $i$, our expressions for the rates are different from those of Weitzman (1998). There is a bias towards the more impatient agents in the consensus equilibrium discount rates. A possible interpretation is as follows. When considering its intertemporal rate of substitution, the group must weigh more the agents with a higher shadow price of the intertemporal budget constraint, i.e. the more impatient members of the group ${ }^{8}$.

As far as asymptotic properties are concerned, we obtain, as in Jouini et al. (2008) that the relevant rate in the long run is given by the lowest individual discount rate. This rate corresponds to the discount rate of the most patient agent (lowest $\rho_{i}$ ) when there is no beliefs heterogeneity, or to the most pessimistic agent (lowest $\mu_{i}$ ) when there is no pure time preference rate heterogeneity and all the agents have the same volatility parameter or to the least confident agent (highest $\sigma_{i}^{2}$ ) when there is no pure time preference rate heterogeneity and all the agents have the same drift parameter. Moreover, the equilibrium approach leads to decreasing discount rates, not only utility discount rates, but also consumption discount rates. As in the

\footnotetext{
${ }^{8}$ Note that optimism/pessimism in the form of a higher/lower $\mu_{i}$ or overconfi dence/doubt in the form of a lower/higher $\sigma_{i}^{2}$ have no impact on the relative weights, but this might be due to the specific logarithmic utility function.
} 
certainty approach of Weitzman (1998), this leads to use lower discount rates for long term projects in a cost-benefit analysis.

In the case of homogeneous beliefs, Equation (3) involves the covariance between $\rho_{i}$ and exp $\rho_{i} t$ as in Lengwiler (2005). Equation (3) also gives us the expression for the consensus utility discount rate $\rho \equiv \sum_{i}^{N} \frac{w_{i} \rho_{i} \exp \left(\rho_{i} t\right)}{\sum_{j}^{N} w_{j} \rho_{j} \exp \left(\rho^{j} t\right)} \rho_{i}$.

Although of the same nature, this expression is slightly different from the one obtained through the Pareto optimality of Gollier and Zeckhauser (2005) or the Benthamite approach of Nocetti et al. (2008). Indeed, our weights in the weighted averages of the $\rho_{i}$ are given by the quantities $w_{i} \rho_{i} \exp \rho_{i} t$ whereas they are given by $\lambda_{i} \exp \rho_{i} t$ in Gollier and Zeckhauser (2005) or Nocetti et al. (2008), where the $\left(\lambda_{i}\right)$ are Pareto weights chosen by the social planner. Notice that this means that our equilibrium approach and the Pareto/Benthamite approach would lead to the same social utility discount rate if the Pareto weights were proportional to $w_{i} \rho_{i}$.

\subsection{Comparison with other formula for the socially efficient discount rate}

The following proposition clarifies the relation between our socially efficient discount rate and the different expressions that have been provided in the literature.

Proposition 2 1. The equilibrium discount rate is lower than the pure time preference weighted arithmetic average of the individual discount rates, i.e.

$$
R_{t} \leq \sum_{i 1}^{N} \frac{w_{i} \rho_{i}}{\sum_{j 1}^{N} w_{j} \rho_{j}} R^{i} \text { and } r_{t} \leq \sum_{i=1}^{N} \frac{w_{i} \rho_{i}}{\sum_{j{ }_{1}{ }_{1} w_{j} \rho_{j}}^{N}} r^{i}
$$

2. If the tastes and beliefs characteristics $\rho_{i}$ and $b_{i} \equiv \mu_{i} \quad \sigma_{i}^{2}$ are independent or if they are comonotonic, i.e. individuals with higher tastes characteristics $\rho_{i}$ have higher beliefs characteristics $b_{i}$, then the equilibrium discount rate is higher than an average of the individual discount rates, i.e.

$$
R_{t} \geq \frac{1}{t} \ln \sum_{i}^{N} w_{i} \exp \quad R^{i} t
$$


3. If the tastes and beliefs characteristics $\rho_{i}$ and $b_{i} \equiv \mu_{i} \quad \sigma_{i}^{2}$ are independent, then the equilibrium marginal discount rate is higher than an average of the individual marginal discount rates, i.e.

$$
r_{t} \geq \sum_{i=1}^{N} \frac{w_{i} \exp r^{i} t}{\sum_{j}^{N} w_{j} \exp r^{j} t} r^{i} .
$$

Equation (4) means that, as expected, the discount rate to use is lower than the simple arithmetic average (with the same weights) of the individual discount rates. Moreover, Equations (5) and (6) imply that, when tastes and beliefs characteristics are independent, our discount rates are higher than those of Weitzman (2001). This is intuitive since our weights are given by the pure time preference rates $\rho_{i}$ hence higher weights are granted to higher individual discount rates.

\section{Specific Distributions and Dominance Prop- erties}

Let us now determine the equilibrium discount rate for specific distributions of the experts discount rates $\left(R^{i}\right)$. Equation (1) as well as Proposition 1 provide the expression of the discount rates $R$ and $r$ as a function of the experts discount rates $\left(R^{i}\right)$ or $\left(r^{i}\right)$.

\subsection{Gaussian and Gamma distributions}

We whall consider continuous sets of experts. It is easy to show that the expression of the discount rates remains the same in the setting with a continuum of agents ${ }^{9}$. The problem is that according to Equations (1) and (2), we need to make extra assumptions on the joint distribution of $\left(\rho_{i}, R^{i}\right)$ in order to determine the discount rates $R$ and $r$.

Consider first the case with homogeneous pure time preference rates $\rho_{i} \quad \rho$, and with a normal distribution $\mathcal{N}\left(m, v^{2}\right)$ on the beliefs parameters

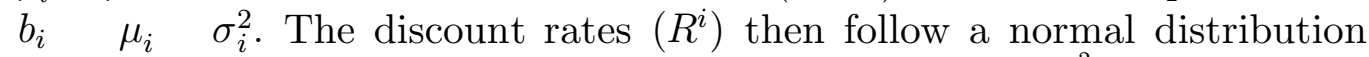
$\mathcal{N}\left(\rho+m, v^{2}\right)$ and we easily obtain that $R_{t} \quad \rho+m \quad \frac{v^{2}}{2} t$. Reinschmidt (2002) obtains a similar formula for the consensus utility discount rate when the individual utility discount rates follow a normal distribution.

\footnotetext{
${ }^{9}$ See Section 4 for a proof in a more general setting.
} 
Suppose now that utility discount rates and beliefs are independently ${ }^{10}$ and gamma distributed. We obtain the following result.

Proposition 3 If pure time preference rates $\rho_{i}$ and beliefs $b_{i} \quad \mu_{i} \quad \sigma_{i}^{2}$ are independently distributed ${ }^{11}$ with $\rho_{i} \sim \gamma\left(\alpha_{1}, \beta_{1}\right)$ and $b_{i} \sim \gamma\left(\alpha_{2}, \beta_{2}\right)$, then

1. $R_{t} \quad \frac{\alpha_{1}+1}{t} \ln \frac{\beta_{1}}{\beta_{1}+t} \quad \frac{\alpha_{2}}{t} \ln \frac{\beta_{2}}{\beta_{2}+t}$ and $r_{t} \quad \frac{\alpha_{1}+1}{\beta_{1}+t}+\frac{\alpha_{2}}{\beta_{2}+t} \quad \frac{m_{1}^{2}+v_{1}^{2}}{m_{1}+t v_{1}^{2}}+\frac{m_{2}^{2}}{m_{2}+t v_{2}}$ where $\left(m_{1}, v_{1}^{2}\right)$ and $\left(m_{2}, v_{2}^{2}\right)$ respectively denote the mean and variance of $\left(\rho_{i}\right)$ and $\left(b_{i}\right)$.

2. If $\beta_{1} \quad \beta_{2}$ then $R^{i} \sim \gamma(\alpha, \beta)$ with $\alpha \quad \alpha_{1}+\alpha_{2}, R_{t} \quad R_{t}^{W}+\frac{1}{t} \ln \left(1+\frac{t}{\beta}\right)$ and $r_{t} \quad \frac{m^{2}+v^{2}}{m+t v^{2}} \quad r_{t}^{W}+\frac{1}{\beta+t}$ where $r_{t}^{W}$ and $R_{t}^{W}$ respectively denote the marginal discount rate and the discount rate obtained through the certainty equivalent approach of Weitzman and where $(m, v)$ denote the mean and variance of $\left(R^{i}\right)$.

A decrease in the mean $m_{2}$ or an increase in the variance $v_{2}^{2}$ of the individual beliefs $\left(b_{i}\right)$ decreases the marginal discount rate $r_{t}$ (hence the discount rate $R_{t}$ ). The same result occurs with a decrease in the mean $m_{1}$ of the individual pure time preference rates $\left(\rho_{i}\right)$. An increase in the variance $v_{1}^{2}$ of the individual pure time preference rates $\left(\rho_{i}\right)$ decreases the marginal discount rate $r_{t}$ for $t$ large enough.

When beliefs and tastes are independent and follow gamma distributions with the same parameter $\beta$, the distribution of the individual discount rates $R^{i}$ or $r^{i}$ is a sufficient statistics for the equilibrium discount rate. As in Weitzman (2001), experts discount rates then follow a Gamma distribution. As shown in the previous section, our equilibrium discount rates are higher than Weitzman (2001)'s discount rates but converge to the same value. A decrease in the mean $m$ of the individual discount rates $\left(R^{i}\right)$ decreases the marginal discount rate and an increase in the variance $v^{2}$ of the individual discount rates $\left(R^{i}\right)$ decreases the marginal discount rate $r_{t}$ for $t$ large enough.

\footnotetext{
${ }^{10}$ We focus on the case of independent distributions as a central case; explicit formulas may also be derived in the case of a given correlation between pure time preference rates and beliefs.

${ }^{11}$ Recall that the density function of a gamma distribution $\gamma(\alpha, \beta)$ is given by $\frac{\beta^{\alpha}}{\Gamma(\alpha)} x^{\alpha}{ }^{1} \exp (-\beta x)$. Its mean $m$ and its variance $v^{2}$ are respectively given by $m=\frac{\alpha}{\beta}$ and $\frac{\alpha}{\beta^{2}}$.
} 


\subsection{Calibration on Weitzman (2001)'s data}

We now calibrate this model with two independent gamma distributions $\rho_{i} \sim \gamma\left(\alpha_{1}, \beta_{1}\right)$ and $b_{i} \sim \gamma\left(\alpha_{2}, \beta_{2}\right)$ on Weitzman (2001)'s data. We impose that $m_{1}+m_{2} \quad m$ and $v_{1}^{2}+v_{2}^{2} \quad v^{2}$ where $m$ and $v^{2}$ respectively denote the mean and the variance of the individual discount rates computed on Weitzman(2001)'s sample. We further impose that $\frac{m_{1}}{v_{1}} \quad \frac{m_{2}}{v_{2}}$ (same ratio between mean and standard deviation for both distributions), which leads to $\alpha_{1} \quad \alpha_{2}$ and $\frac{\beta_{1}}{\beta_{2}} \quad \frac{m_{2}}{m_{1}} \equiv \lambda$, for some positive $\lambda$. Note that for $\lambda \quad 1$, we get $\left(\alpha_{1}, \beta_{1}, \alpha_{2}, \beta_{2}\right) \quad\left(\frac{m^{2}}{2 v^{2}}, \frac{m}{v^{2}}, \frac{m^{2}}{2 v^{2}}, \frac{m}{v^{2}}\right)$, which corresponds to the calibration in Weitzman (2001). We have then a family of stastistical models that contains Weitzman (2001)'s statistical model and we maximize the loglikelihood with respect to the parameter $\lambda$ to choose the best calibration. We obtain $\lambda \quad 0.4116$ hence $\left(\alpha_{1}, \beta_{1}, \alpha_{2}, \beta_{2}\right) \quad(1.043,89.454,1.043,36.819)$ and $\left(m_{1}, v_{1}^{2}, m_{2}, v_{2}^{2}\right) \quad\left(1.16 \times 10^{2}, 1.30 \times 10^{4}, 2.83 \times 10^{2}, 7.69 \times 10^{4}\right)$. To summarise, the best calibration corresponds to a gamma distribution on the individual pure time preference rates with an average time preference rate among experts equal to $1.16 \%$ and a median equal to $0.67 \%$ and a gamma distribution on the individual beliefs with an average belief parameter (about the growth of the economy) equal to $2.83 \%$ and a median equal to $2 \%$. More precisely, the belief parameter $b \quad \mu \quad \sigma^{2}$ can be interpreted as a risk adjusted growth rate. The values we obtain are then reasonable values for both an average pure time preference rate and an average risk-adjusted growth rate. Stern report considers values for the pure time preference rate (utility discount rate) between 0.1 and 1.5 and values for the growth rate ranging from 0 per cent to 6 per cent. Arrow (1995) states that the pure time preference rate should be about $1 \%$ and surveying the evidence, the HM Treasury's Green Book (2003) suggests a long run growth rate of 2.1 per cent.

Figure 1 represents the log-likelihood as a function of $\lambda$. Figure 2 represents the distribution of the individual discount rates for the parameter $\lambda$ that maximizes the $\log$-likelihood $(\lambda \quad 0.4116)$ as well as the empirical distribution and Weitzman (2001)'s distribution. Figure 3 represents the corresponding marginal discount rate curve and compares it to the discount rate curve of Weitzman (2001). Table 1 presents the corresponding recommended sliding-scale discount rates: Immediate Future about 5 per cent; Near Future about 4 percent; Medium Future about 3 percent; Distant Future about 1.5 per cent and Far-Distant Future about 0 per cent. 


\subsection{Dominance properties}

In the specific setting of Gamma distributions considered in Proposition 3, we have seen the impact of an increase in the mean and in the variance of the distribution of individual discount rates on the socially efficient discount rates. The impact was the same for the average and for the marginal discount rates. We now analyze in a more general setting which shifts on the distribution of individual discount rates have a clear impact on the socially efficient discount rates. We consider First and Second Stochastic Dominance shifts, as defined in e.g. Rothschild and Stiglitz (1970). In order to obtain a clear impact on the socially efficient marginal discount rate $r$, we also consider Monotone Likelihood Ratio dominance (MLR) shifts. This concept of dominance has been studied by Landsberger and Meilijson (1990) and is defined as follows: a random variable $Y$ dominates a random variable $X$, if $X$ and $Y$ have densities with respect to some dominating measure $\nu$ such that $f_{X}(x) f_{Y}(y) \leq f_{X}(y) f_{Y}(x)$ for all $y \leq x$ (roughly speaking, the ratio $\frac{f_{Y}}{f_{X}}$ is nondecreasing).

Proposition 4 1. If all the agents have the same time preference rate $\rho_{i}$, then a FSD (resp. SSD) shift in the distribution of $\left(R^{i}\right)$ increases the discount rate $R_{t}$ for all horizons.

2. If all the agents have the same time preference $\rho_{i}$, then a MLR shift in the distribution of the $\left(r^{i}\right)$ increases the marginal discount rate $r_{t}$ for all horizons.

3. If all the agents have the same beliefs, then a MLR shift in the distribution of the $\left(R^{i}\right)$ increases the discount rate $R_{t}$ for all horizons.

Proposition 4 makes it clear which concepts of dominance (corresponding to the notions of pessimism, doubt, patience, or heterogeneity) one should consider in order to have a clear impact on the discount rates. Roughly speaking, it means that a country where experts are more pessimistic and/or exhibit more doubt about future growth and/or have lower pure time preference rates (more patient or more altruistic with respect to future generations) should apply a lower discount rate for cost-benefit analysis. More heterogeneity in experts beliefs about future growth rates also leads to lower discount rates. 
More precisely, suppose that in one population, say (A), we have three equally large groups ${ }^{12}$ with discount rates of $2 \%, 3 \%$ and $4 \%$. In a second population (B), there are also three groups with the same anticipated growth rates but their proportion in the population is $\frac{1}{2}, \frac{1}{6}$ and $\frac{1}{3}$. Population (B) is more pessimistic than population (A) (in the sense of the FSD) and the discount rate to apply is lower for (B). In a third population (C), there are three groups with anticipated growth rates $1 \%, 3 \%$ and $5 \%$ and their proportion in the population is $\frac{1}{10}, \frac{8}{10}$ and $\frac{1}{10}$. Populations (A) and (C) have the same average level of pessimism but population $(\mathrm{C})$ is more heterogeneous (in the sense of the SSD) than population (A) and the discount rate to apply is lower for $(\mathrm{C})$. Let us assume now that experts provide forecasts with a $95 \%$ confidence interval. Let us assume that these intervals in population (A) are given by $[1.5,2.5],[2.5,3.5]$ and $[3.5,4.5]$ while in a fourth population (D) also with three equally large groups, these intervals are given by $[1 ; 3],[2 ; 4]$ and $[3 ; 4]$. There is more doubt in population (D) and the discount rate to apply is then lower for (D). The MLR (monotone likelihood ratio) dominance is stronger than the FSD dominance. Let us consider two populations (E) and (F). In population (E), there are three equally large groups of experts with pure time preference rates respectively equal to $0.5 \%, 1 \%$ and $1.5 \%$. In population $(\mathrm{F})$ there are also three groups with the same pure time preference rates but with proportions in the population respectively equal to $w_{1}, w_{2}$ and $w_{3}$. The population (E) is more patient (in the sense of the MLR) if $w_{3}<w_{2}<w_{1}$. In this case, the discount rate to apply for cost-benefit analysis is lower for population (E).

\section{Extensions}

In this section, we examine two possible extensions: more general subjective and objective distributions for aggregate consumption and time dependent pure time preference rates.

\subsection{General distributions for aggregate consumption}

We first show that Proposition 1 remains valid in a very general complete markets Arrow-Debreu setting. Time can be continuous or discrete. We

\footnotetext{
${ }^{12}$ In this paragraph, for simplicity, we refer to groups and their size instead of experts and the size of the population that they represent.
} 
allow for a finite number or a continuum of agents. For this purpose, the set of agents is represented by a measured space $([0,1], \nu)$. Furthermore we do not need to assume specific individual distributions for aggregate consumption. It suffices to assume that agent $i$ has a probability measure $Q_{t}^{i}$ that represents the distribution of date $t$ aggregate consumption from agent $i$ point of view. As in previous sections, agent $i$ has a pure time preference rate $\rho_{i}$, a share of total wealth $w_{i}$ and a log-utility.

Proposition 5 Let us consider a model with a measured space $([0,1], \nu)$ of log-utility agents that have pure time preference rates $\left(\rho_{i}\right)$, wealth shares $\left(w_{i}\right)$ and date $t$ probability measures $Q_{t}^{i}$. We assume that all these probabilities are equivalent, i.e. the agents agree on the events of zero probability. The equilibrium discount rate is then given by

$$
R_{t} \equiv \frac{1}{t} \ln \int_{0}^{1} \frac{w_{i} \rho_{i}}{\int_{0}^{1} w_{j} \rho_{j} d \nu(j)} \exp \left(R_{t}^{i} t\right) d \nu(i)
$$

where $R_{t}^{i}$ is the equilibrium discount rate that would prevail if the economy was made of agent $i$ only.

In such a general setting, the equilibrium discount rate is still a weighted average of the individual discount rates, and as previously, the weights are proportional to $w_{i} \rho_{i}$. The only difference with the setting of Equation (1) is the fact that the individual discount rates $R_{t}^{i}$ may depend upon $^{13} t$.

\subsection{Time dependent pure time preference rates}

It is also easy to adapt our approach to the case with time-dependent pure time preference rates $\left(\rho_{i}(t)\right)$. We then have $R_{t}^{i} \quad \frac{1}{t} \int_{0}^{t} \rho_{i}(s) d s+\mu_{i} \quad \sigma_{i}^{2}$ and

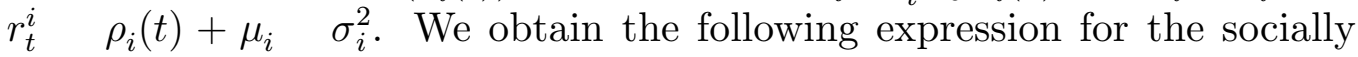
efficient discount rate.

Proposition 6 If agents have time-dependent positive pure time preference rates $\left(\rho_{i}(t)\right)$, wealth shares $\left(w_{i}\right)$ and date $t$ distributions for aggregate

\footnotetext{
${ }^{13}$ In particular, if aggregate consumption is lognormal, then the individual discount rates are time independent, and the expression for the socially efficient discount rate is the same as in Equation (1), replacing sums by integrals.
} 
consumption $\ln \mathcal{N}\left(\left(\mu_{i} \quad \frac{1}{2} \sigma_{i}^{2}\right) t, \sigma_{i}^{2} t\right)$, the equilibrium discount rates are given by

$$
\begin{aligned}
R_{t} & \equiv \frac{1}{t} \ln \sum_{i 1}^{N} \frac{w_{i} \bar{\rho}_{i}}{\sum_{i}^{N} w_{j} \bar{\rho}_{j}} \exp \left(R_{t}^{i} t\right) \\
r_{t} & \equiv \sum_{i}^{N} \frac{w_{i} \bar{\rho}_{i} \exp \left(\int_{0}^{t} r_{s}^{i} d s\right)}{\sum_{i}^{N}{ }_{1} w_{j} \bar{\rho}_{j} \exp \left(\int_{0}^{t} r_{s}^{i} d s\right)} r_{t}^{i}
\end{aligned}
$$

for $R_{t}^{i} \quad \frac{1}{t} \int_{0}^{t} \rho_{i}(s) d s+\mu_{i} \quad \sigma_{i}^{2}, r_{t}^{i} \quad \rho_{i}(t)+\mu_{i} \quad \sigma_{i}^{2}$ and $\bar{\rho}_{i} \quad\left(\int_{0}^{\infty} \exp \left(\int_{0}^{t} \rho_{i}(s) d s\right) d t\right)^{1}$.

If the pure time preference rates $\left(\rho_{i}(t)\right)$ are decreasing with $t$, then the discount rates $R_{t}$ and $r_{t}$ are also decreasing with $t$ and we have

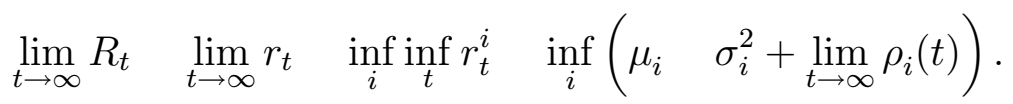

\section{Conclusion}

In this paper, we propose an equilibrium approach to aggregate the discount rates proposed by experts into a consensus discount rate. We emphasize that our approach enables to deal with consumption discount rates and not only with utility discount rates (pure time preference rates).

We start with the recognition that divergence among experts on what the discount rate should be is rooted in fundamental differences of opinion about inter-generational equity as well as about future growth of the quantity of available consumption. This enables us to translate the problem of aggregating experts discount rates into a problem of aggregating heterogeneous beliefs and time preference rates. We can then use the techniques of Jouini et al. (2008) in order to obtain the expression of the socially efficient discount rate. The equilibrium discount rate is a weighted average of the experts proposed discount rates, in which more impatient experts are more heavily weighted; the equilibrium discount rate is decreasing and converges to the lowest expert discount rate, which does not necessarily correspond to the discount rate of the most 'patient' expert.

We show that the equilibrium discount rate is higher than Weitzman (1998)'s certainty equivalent discount rate for all horizon. More divergence of opinion about future growth rates among experts (in the form of second stochastic dominated shifts) leads to lower discount rates for all horizons. 
More doubt (larger confidence intervals) as well as more pessimism (in the form of first stochastic or monotone likelihood ratio dominated shifts) in experts' forecasts also leads to lower discount rates.

We calibrate the model on Weitzman (2001)'s data. We show that the very wide spread of opinion on discount rates makes the effective equilibrium discount rate decline significantly over time from 5 per cent per annum for Immediate Future to 0 per cent per annum for Far-Distant Future.

\section{References}

[1] Arrow, K.J., 1995. Inter-generational equity and the rate of discount in long-term social investment. Paper at IEA World Congress (December), available at http://wwwecon.stanford.edu/faculty/workp/swp97005.pdf.

[2] Groom, B., Hepburn, C., Koundouri, P., and D. Pearce, 2005. Declining Discount Rates : the Long and the Short of it. Environmental and Resource Economics, 445-493.

[3] Gollier, C., 2004. Maximizing the expected net future value as an alternative strategy to gamma discounting. Finance Research Letters, 1, 85-89.

[4] Gollier, C., and R. Zeckhauser, 2005. Aggregation of heterogeneous time preferences. Journal of Political Economy, 113, 4, 878-898.

[5] HM Treasury, 2003. The Green Book Appraisal and Evaluation in Central Government. HM Treasury, London.

[6] Jouini, E., Marin, J.-M., and C. Napp, 2008. Discounting and divergence of opinion. Working Paper, available at http://papers.ssrn.com/sol3/papers.cfm?abstract_id 915380\#PaperDownload

[7] Landsberger, M., and I. Meilijson, 1990. Demand for risky financial assets: A portfolio analysis. Journal of Economic Theory, 50, 204-213.

[8] Lengwiler, Y., 2005. Heterogeneous patience and the term structure of real interest rates. American Economic Review, 95, 890-896. 
[9] Nocetti, D., Jouini, E., and C. Napp, 2008. Properties of the Social Discount Rate in a Benthamite Framework with Heterogeneous Degrees of Impatience. Management Science, To appear.

[10] Nordhaus, W., 2007. A Review of the Stern Review on the Economics of Climate Change. Journal of Economic Literature, 45(3), 686-702.

[11] Ramsey, F., 1928. A mathematical theory of savings. Economic Journal, 38, 543-559.

[12] Reinschmidt, K.F., 2002. Aggregate Social Discount Rate Derived from Individual Discount Rates. Management Science, 48 (2), 307-312.

[13] Rothschild, M. and J.E. Stiglitz, 1970. Increasing risk: I. A definition. Journal of Economic Theory, 2, 225-243

[14] Rubinstein, M., 1975. The strong case for the generalized Logarithmic Utility Model as the Premier Model of Financial Markets. The Journal of Finance, 31, 2, 551-571.

[15] Weitzman, M., 1998. Why the Far-Distant Future Should Be Discounted at its Lowest Possible Rate. Journal of Environmental Economics and Management, 36, 201-208.

[16] Weitzman, M., 2001. Gamma Discounting. The American Economic Review, 91, 1, 260-271.

[17] Weitzman, M., 2007. A Review of the Stern Review on the Economics of Climate Change. Journal of Economic Literature, 45(3), 703-724.

\section{Appendix}

\section{Derivation of the extended Ramsey equation}

At the equilibrium, the date $t$ state price density $q_{t}$ is given by

$$
q_{t} \quad \exp (\rho t) u^{\prime}\left(e_{t}\right)
$$

and the discount rate is given by

$$
R \quad \frac{1}{t} \ln E\left[q_{t}\right] \quad \rho \quad \frac{1}{t} \ln E\left[e_{t}^{\eta}\right] .
$$


The random variable $e_{t}{ }^{\eta}$ follows a log normal distribution with parameters $\eta\left(\begin{array}{ll}\mu & \frac{1}{2} \sigma^{2}\end{array}\right) t$ and $\sigma^{2} \eta^{2} t$. We then have $\ln E\left[\begin{array}{ll}c^{\frac{1}{\eta}} \\ \quad t(\eta \mu & \frac{1}{2} \eta(1+\eta) \sigma^{2}\end{array}\right)$ and

$$
R \quad \rho+\eta \mu \quad \frac{1}{2} \eta(1+\eta) \sigma^{2} .
$$

Proof of Equation 1 and of Proposition 1

We first prove that $A_{t} \quad \sum_{i}^{N}{ }_{1} \gamma_{i} \exp { }^{R^{i} t} \quad \sum_{i}^{N}{ }_{1} \gamma_{i} \exp { }^{r^{i} t}$, with $\gamma_{i}$ $\frac{w_{i} \rho_{i}}{\sum_{j}^{N} w_{j} \rho_{j}}$. Let us denote by $q_{t}$ the date $t$ state-price density (with respect to $P$ ) and by $y_{t}^{i}$ the consumption of group $i$. We let $M^{i} \equiv \frac{f_{i}}{f}\left(e_{t}\right)$ denote the density of agenti's belief with respect to the true probability. Each group maximizes its aggregate utility $\int_{0}^{\infty} \exp \left(\rho_{i} t\right) E\left[M_{t}^{i} \log y_{t}^{i}\right] d t$ under its budget constraint $\int_{0}^{\infty} E^{P}\left[q_{t} y_{t}^{i}\right] d t \leq w_{i} \int_{0}^{\infty} E^{P}\left[q_{t} e_{t}\right] d t$. This leads to the following Euler conditions

$$
\frac{1}{\lambda_{i}} \exp \left(\rho_{i} t\right) M_{t}^{i} \frac{1}{y_{t}^{i}} \quad q_{t} \quad \text { for all } i
$$

for some positive Lagrange multipliers $\left(\lambda_{i}\right)$

We have then

$$
\frac{1}{\lambda_{i}} \exp \left(\rho_{i} t\right) M_{t}^{i} \frac{1}{q_{t}} \quad y_{t}^{i}
$$

and summing all these equations leads to

$$
q_{t} \quad \sum_{i=1}^{N} \frac{1}{\lambda_{i}} \exp \left(\rho_{i} t\right) M_{t}^{i} \frac{1}{e_{t}} .
$$

Now, in our setting, exp $r^{i t} \quad \exp R^{R^{i} t} \quad E\left[\exp \left(\rho_{i} t\right) M_{t}^{i} \frac{1}{e_{t}}\right]$, hence

$$
A_{t} \quad E\left[q_{t}\right] \quad \sum_{i}^{N} \frac{1}{\lambda_{i}} \exp ^{r^{i} t} \quad \sum_{i}^{N} \frac{1}{\lambda_{i}} \exp ^{R^{i} t} .
$$

It remains to determine the equilibrium weights $\frac{1}{\lambda_{i}}$. From the Euler and budget conditions we have

$$
\int_{0}^{\infty} E^{P}\left[q_{t} y_{t}^{i}\right] d t \quad \frac{1}{\lambda_{i} \rho_{i}} \quad w_{i} \int_{0}^{\infty} E^{P}\left[q_{t} e_{t}\right] d t
$$

which leads to

$$
\frac{1}{\lambda_{i}} \quad \frac{\rho_{i} w_{i}}{\sum_{j}^{N} \rho_{j} \rho_{j} w_{j}} .
$$


We easily deduce Equations (1), (2) as well as Equation (3).

As far as the monotony of $r$ is concerned, we have $r_{t} \equiv \sum_{i}^{N}{ }_{1} \frac{w_{i} \rho_{i} \exp \left(r^{i} t\right)}{\sum_{j}^{N} w_{j} \rho_{j} \exp \left(r^{j} t\right)} r^{i}$, then $\frac{d r_{t}}{d t} \quad \frac{\left(\sum_{i}^{N} w_{i} \rho_{i} \exp \left(r^{i} t\right) r^{i}\right)^{2}}{\left(\sum_{i 1}^{N} w_{i} \rho_{i} \exp \left(r^{i} t\right)\right)^{2}} \quad \sum_{i}^{N} 1 \frac{w_{i} \rho_{i} \exp \left(r^{i} t\right)}{\sum_{j}^{N} w_{j} \rho_{j} \exp \left(r^{j} t\right)}\left(r^{i}\right)^{2}$. Let us consider $P_{\rho \exp }$ the probability measure whose weights are proportional to by $w_{i} \rho_{i} \exp \left(r^{i} t\right)$. We have $\frac{d r_{t}}{d t} \quad E^{P_{\rho \exp }}[r]^{2} \quad E^{P_{\rho \exp }}\left[r^{2}\right] \leq 0$. The marginal discount rate $r_{t}$ decreases then with $t$.

As far as the asymptotic behavior of the marginal discount rate $r$ is concerned, let $r^{i^{*}} \equiv \inf _{i} r^{i}$ and let $j$ be such that $r^{j} / r^{i^{*}}$, then the relative weight of $r^{j}$ in Equation (2) converges to zero and $r_{t} \rightarrow_{t \rightarrow \infty} r^{i^{*}}$.

Proof of Proposition 2

1. According to Proposition 1, we have

$$
R_{t} \equiv \frac{1}{t} \ln A_{t}
$$

where $A_{t}$ is an arithmetic average of the exp $R^{i} t$. Since the arithmetic average is larger than the geometric average, we have

$$
\sum_{i 1}^{N} \frac{w_{i} \rho_{i}}{\sum_{j 1}^{N} w_{j} \rho_{j}} \exp \left(R^{i} t\right) \geq \exp \sum_{i=1}^{N} \frac{w_{i} \rho_{i}}{\sum_{j 1}^{N} w_{j} \rho_{j}} R^{i} t .
$$

Hence,

$$
R_{t} \leq \sum_{i}^{N} \frac{w_{i} \rho_{i}}{\sum_{j}^{N}{ }_{1} w_{j} \rho_{j}} R^{i}
$$

We have

$$
\begin{aligned}
r_{t} \equiv & \sum_{i=1}^{N} \frac{w_{i} \rho_{i} \exp \left(r^{i} t\right)}{\sum_{j}^{N} w_{j} \rho_{j} \exp \left(r^{j} t\right)} r^{i} \\
& \frac{E^{P_{\rho}}[\exp (r t) r]}{E^{P_{\rho}}[\exp (r t)]}
\end{aligned}
$$

where $P_{\rho}$ has weights $\frac{w_{i} \rho_{i}}{\sum_{j}^{N} w_{j} \rho_{j}}$. Since $\exp \left(r^{i} t\right)$ decreases with $r^{i}$, we have $E^{P_{\rho}}\left[\exp (r t) r^{i}\right] \leq E^{P_{\rho}}[r] E^{P_{\rho}}[\exp (r t)]$. Hence

$$
r_{t} \leq E^{P_{\rho}}[r] \quad \sum_{i}^{N} \frac{w_{i} \rho_{i}}{\sum_{j}^{N} w_{j} w_{j}} r^{i}
$$


2. Let us denote by $P_{w}$ the probability measure with weights $w_{i}$. Since $\rho_{i}$ and $b_{i} \equiv \mu_{i} \quad \sigma_{i}^{2}$ are independent, we have

$$
\begin{aligned}
\exp \left(R_{t} t\right) & \frac{E^{P_{w}}[\rho \exp (\rho t) \exp (b t)]}{E^{P_{w}}[\rho]}, \\
& \frac{E^{P_{w}}[\rho \exp (\rho t)] E^{P_{w}}[\exp (b t)]}{E^{P_{w}}[\rho]} .
\end{aligned}
$$

Now, since $\rho_{i}$ and $\exp \left(\rho_{i} t\right)$ are anticomonotonic, we have

$$
E^{P_{w}}[\rho \exp (\rho t)] \leq E^{P_{w}}[\rho] E^{P_{w}}[\exp (\rho t)]
$$

which gives

$$
\begin{aligned}
\exp \quad R_{t} t & \geq E^{P_{w}}[\exp (\rho t)] E^{P_{w}}[\exp (b t)] \\
& \geq E^{P_{w}}[\exp (r t)]
\end{aligned}
$$

3. We have

$$
r_{t} \frac{E^{P_{\exp }}\left[\rho^{2}\right]+E^{P_{\exp }}[\rho b]}{E^{P_{\exp }}[\rho]} .
$$

where $P_{\exp }$ denotes the probability measure whose weights are proportional to $w_{i} \exp \left(r^{i} t\right)$. We have then

$$
\begin{aligned}
r_{t} & \geq \frac{E^{P_{\exp }}[\rho]^{2}+E^{P_{\exp }}[\rho b]}{E^{P_{\exp }}[\rho]}, \\
& \geq E^{P_{w}}[\rho \exp (\rho t)] \frac{E^{P_{w}}[\rho \exp (\rho t)] E^{P_{w}}[\exp (\quad b t)]+E^{P_{w}}[\exp (\quad b t) b] E^{P_{w}}[\exp (\rho t)]}{E^{P_{w}}[\exp (\rho t)] E^{P_{w}}[\rho \exp (r t)]} \\
& \geq \frac{E^{P_{w}}[(\rho+b) \exp ((\rho+b) t)]}{E^{P_{w}}[\exp (\rho t)] E^{P_{w}}[\exp (b t)]} \\
& \geq \sum_{i}^{N} \frac{w_{i} \exp \left(r^{i} t\right)}{\sum_{j}^{N}{ }_{1} w_{j} \exp \left(r^{j} t\right)} r^{i} .
\end{aligned}
$$

Proof of Proposition 3

If pure time preference rates $\rho_{i}$ and beliefs $b_{i} \quad \mu_{i} \quad \sigma_{i}^{2}$ are independent and are distributed as follows $\rho_{i} \sim \gamma\left(\alpha_{1}, \beta_{1}\right)$ and $b_{i} \sim \gamma\left(\alpha_{2}, \beta_{2}\right)$, then 


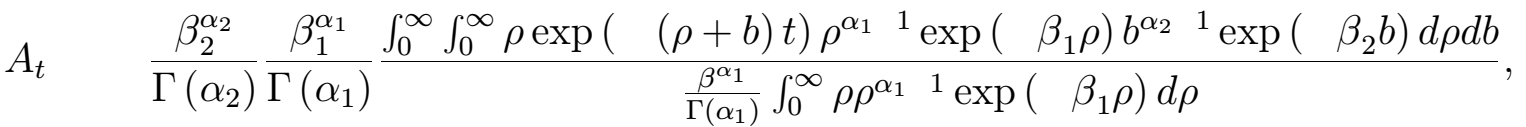

$$
\begin{aligned}
& \frac{\beta_{2}^{\alpha_{2}}}{\Gamma\left(\alpha_{2}\right)} \frac{\left(\int_{0}^{\infty} \exp \left(\rho\left(\beta_{1}+t\right)\right) \rho^{\alpha_{1}} d \rho\right)\left(\int_{0}^{\infty} \exp \left(b\left(\beta_{2}+t\right)\right) b^{\alpha_{2}}{ }^{1} d b\right)}{\int_{0}^{\infty} \rho^{\alpha_{1}} \exp \left(\beta_{1} \rho\right) d \rho}, \\
& \left(\frac{\beta_{1}}{\beta_{1}+t}\right)^{1+\alpha_{1}}\left(\frac{\beta_{2}}{\beta_{2}+t}\right)^{\alpha_{2}} \text {. }
\end{aligned}
$$

The results on the expression of $R$ and $r$ are then easily derived.

\section{Proof of Proposition 4}

1. Let us assume that all the agents have the same $\rho_{i}$, we have then

$$
R_{t} \equiv \frac{1}{t} \ln E^{P_{w}}[\exp \quad R t]
$$

where $P_{w}$ is defined as in the proof of Proposition. For a given $t$, the function $R \rightarrow \exp R t$ is decreasing (and convex) and, by definition, a FSD (resp. SSD) shift in the distribution of $\left(R^{i}\right)$ decreases the value of $E^{P_{w}}[\exp R t]$ and increases $R_{t}$.

2. We still assume that all the agents have the same $\rho_{i}$, we have then

$$
r_{t} \frac{E^{P_{w}}[r \exp (r t)]}{E^{P_{w}}[\exp (r t)]} .
$$

Let us consider $P_{w}^{1}$ and $P_{w}^{2}$, two distributions such that $P_{w}^{2} \succeq_{M L R} P_{w}^{1}$. By definition, the density $\phi \quad \frac{d P_{w}^{2}}{d P_{w}^{1}}$ is nondecreasing in $r$ (in other words $i \rightarrow \phi^{i}$ and $i \rightarrow r^{i}$ are comonotonic). We have then, $\frac{E^{P_{w}^{2}[r \exp r t]}}{E^{P_{w}^{2}}[\exp (r t)]} \quad \frac{E^{P_{w}^{1}}[\phi r \exp r t]}{E^{P_{w}^{1}}[\phi \exp (r t)]}$ $\frac{E^{Q_{\exp }[\phi r]}}{E^{\exp [\phi]}}$ where $Q_{\exp }$ is defined by a density with respect to $P_{w}^{1}$ equal (up to a constant) to $\exp (r t)$. Since $\phi$ is nondecreasing in $r$, we have

$$
E^{Q_{\exp }}[\phi r] \geq E^{Q_{\exp }}[\phi] E^{Q_{\exp }}[r],
$$

hence

$$
\begin{aligned}
\frac{E^{P_{w}^{2}}[r \exp r t]}{E^{P_{w}^{2}}[\exp (r t)]} & \geq E^{Q_{\exp }[r]} \\
& \left.\geq \frac{E^{P_{w}^{1}}[r \exp r}{E^{P_{w}^{1}}[\exp r}\right]
\end{aligned}
$$


3. If we now assume that all the agents have the same belief, we have

$$
R_{t} \equiv \frac{1}{t} \ln \frac{E^{P_{w}}[\rho \exp \rho t]}{E^{P_{w}}[\rho]} .
$$

Let us consider $P_{w}^{1}$ and $P_{w}^{2}$, two distributions such that $P_{w}^{2} \succeq_{M L R} P_{w}^{1}$. We have then, $\frac{E^{P_{w}^{2}}[\rho \exp \rho t]}{E^{P_{w}^{2}[\rho]}} \quad \frac{E^{P_{w}^{1}}[\phi \rho \exp }{E_{w}^{1}[\phi \rho]} \quad \frac{E^{Q \rho}[\phi \exp \rho t]}{E^{Q \rho \rho}[\phi]}$ where $\phi \quad \frac{d P_{w}^{2}}{d P_{w}^{1}}$ and where $Q_{\rho}$ is defined by a density with respect to $P_{w}^{1}$ equal (up to a constant) to $\rho$. Since $\phi$ is nondecreasing in $r$ and then nonincreasing in exp $\rho t$, we have

$$
E^{Q_{\rho}}\left[\begin{array}{ll}
\phi \exp & \rho t
\end{array}\right] \leq E^{Q_{\rho}}[\phi] E^{Q_{\rho}}[\exp \quad \rho t],
$$

hence

$$
\begin{aligned}
\frac{E^{P_{w}^{2}}\left[\begin{array}{ll}
\rho \exp & \rho t
\end{array}\right]}{E^{P_{w}^{2}}[\rho]} & \leq E^{Q_{\rho}}\left[\begin{array}{ll}
\exp & \rho t]
\end{array}\right. \\
& \leq \frac{E^{P_{w}^{1}}\left[\begin{array}{ll}
\rho \exp & \rho t
\end{array}\right]}{E^{P_{w}^{1}}[\rho]}
\end{aligned}
$$

\section{Proof of Proposition 5}

Let $M_{t}^{i}$ denote the density of $Q_{t}^{i}$ with respect to a given probability $P$ equivalent to all the probability measures $Q_{t}^{i}$. Let us denote by $q_{t}$ the date $t$ state-price density (with respect to $P$ ) and by $y_{t}^{i}$ the consumption of group $i$. Each group maximizes its aggregate utility $\int_{0}^{\infty} \exp \left(\rho_{i} t\right) E^{Q_{t}^{i}}\left[\frac{1}{y_{t}^{i}}\right] d t$ under its budget constraint $\int_{0}^{\infty} E^{P}\left[q_{t} y_{t}^{i}\right] d t \leq w_{i} \int_{0}^{\infty} E^{P}\left[q_{t} e_{t}\right] d t$. This leads to the following Euler condition

$$
\frac{1}{\lambda_{i}} \exp \left(\rho_{i} t\right) M_{t}^{i} \frac{1}{y_{t}^{i}} \quad q_{t}
$$

We have then

$$
\frac{1}{\lambda_{i}} \exp \left(\rho_{i} t\right) M_{t}^{i} \frac{1}{q_{t}} \quad y_{t}^{i}
$$

and summing all these equations leads to

$$
q_{t} \quad \sum_{i=1}^{N} \frac{1}{\lambda_{i}} \exp \left(\rho_{i} t\right) M_{t}^{i} \frac{1}{e_{t}} .
$$

Now in an economy made of group $i$ only, we would have 


$$
\exp \left(\rho_{i} t\right) M_{t}^{i} \frac{1}{e_{t}} \quad q_{t}^{i}
$$

and

$$
r_{t}^{i} \quad \frac{1}{t} \ln E\left[q_{t}^{i}\right] .
$$

If all prices are expressed in terms of today's consumption units, we have $q_{0} \quad 1$ and $\sum_{i}^{N} \frac{1}{\lambda_{i}} \quad 1$ which leads to

$$
q_{t} \quad \sum_{i}^{N} \frac{1}{\lambda_{i}} q_{t}^{i}
$$

hence

$$
A_{t} \quad \sum_{i}^{N} \frac{1}{\lambda_{i}} A_{t}^{i} .
$$

It remains to determine the equilibrium weights $\frac{1}{\lambda_{i}}$. From the Euler conditions and budget conditions we have

$$
\int_{0}^{\infty} E^{P}\left[q_{t} y_{t}^{i}\right] d t \quad \frac{1}{\lambda_{i} \rho_{i}} \quad w_{i} \int_{0}^{\infty} E^{P}\left[q_{t} e_{t}\right] d t
$$

which leads to

$$
\frac{1}{\lambda_{i}} \quad \frac{\rho_{i} w_{i}}{\sum_{j}^{N}{ }_{1} \rho_{j} w_{j}}
$$

and $q_{t} \quad \sum_{i}^{N}{ }_{1} \frac{\rho_{i} w_{i}}{\sum_{j}^{N} \rho_{j} w_{j}} q_{t}^{i}$. Since $A_{t} \quad E\left[q_{t}\right]$, we have

$$
A_{t} \quad \sum_{i}^{N} \frac{\rho_{i} w_{i}}{\sum_{j}^{N}{ }_{1} \rho_{j} w_{j}} A_{t}^{i}
$$

hence Equation (7).

Proof of Proposition 6 It is easy to see that the formulas in the proof of Proposition 5 above have to be adapted as follows

$$
\begin{aligned}
q_{t} & \frac{1}{\lambda_{i}} \exp \left(\quad \int_{0}^{t} \rho_{i}(s) d s\right) M_{t}^{i} \frac{1}{y_{t}^{i}} \\
\int_{0}^{\infty} E^{P}\left[q_{t} y_{t}^{i}\right] d t & \int_{0}^{\infty} \frac{1}{\lambda_{i}} \exp \left(\int_{0}^{t} \rho_{i}(s) d s\right) d t
\end{aligned}
$$


The same steps as above lead to

$$
A_{t} \quad \sum_{i=1}^{N} \frac{\bar{\rho}_{i} w_{i}}{\sum_{j}^{N}{ }_{1} \bar{\rho}_{j} w_{j}} A_{t}^{i}
$$

with $\bar{\rho}_{i} \quad\left(\int_{0}^{\infty} \exp \left(\int_{0}^{t} \rho_{i}(s) d s\right) d t\right)^{1}$. 


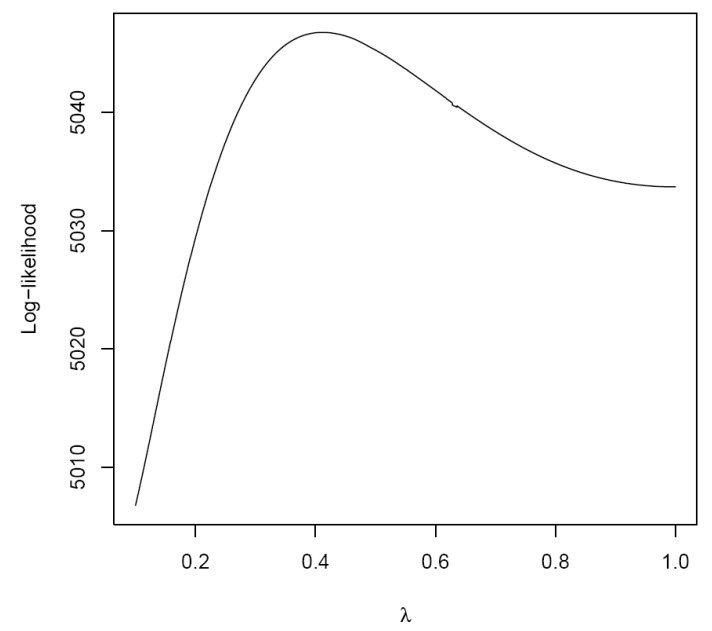

Figure 1: We calibrate a model with two independent gamma distributions (tastes and beliefs) on Weitzman (2001)'s data. We assume that the two distributions are homothetic (the first one is obtained from the second one through a change of variable $x \rightarrow \lambda x$ where $\lambda$ is a given parameter) and we calibrate the model in order to fit the mean and the variance of the empirical distribution. We have then a family of stastical models that contains Weitzman (2001)'s statistical model (it corresponds to $\lambda=1$ ) and we maximize the log-likelihood with respect to the parameter $\lambda$ to choose the best calibration. We obtain $\lambda=0.4116$. 


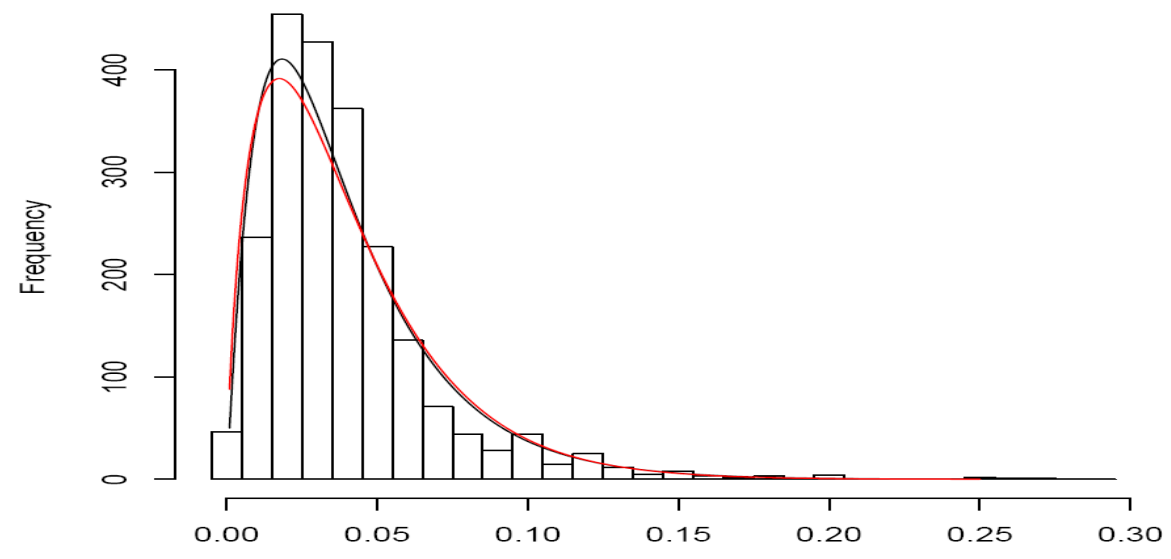

Figure 2: This figure represents the distribution of the individual discount rates for the value $\lambda=0.4116$ that maximizes the log-likelihood (upper curve) as well as the empirical distribution and Weitzman (2001)'s distribution (lower curve). Our distribution corresponds to the sum of two independent gamma distributions with parameters $\left(\alpha_{1}, \beta_{1}\right)$ and $\left(\alpha_{2}, \beta_{2}\right)$ given by $\left(\alpha_{1}, \beta_{1}, \alpha_{2}, \beta_{2}\right)=(1.04,89.45,1.04,36.82)$. These parameters correspond to mean and variance levels given by $\left(m_{1}, v_{1}^{2}, m_{2}, v_{2}^{2}\right)=\left(1.16 \times 10^{-2}, 1.30 \times 10^{-4}, 2.83 \times 10^{-2}, 7.69 \times 10^{-4}\right)$. Weitzman's distribution corresponds to a gamma distribution with parameters $(1.78,44.44)$. All represented distributions have the same mean and variance levels $\left(m, v^{2}\right)=\left(4 \times 10^{-2}, 9 \times\right.$ $\left.10^{-4}\right)$. 


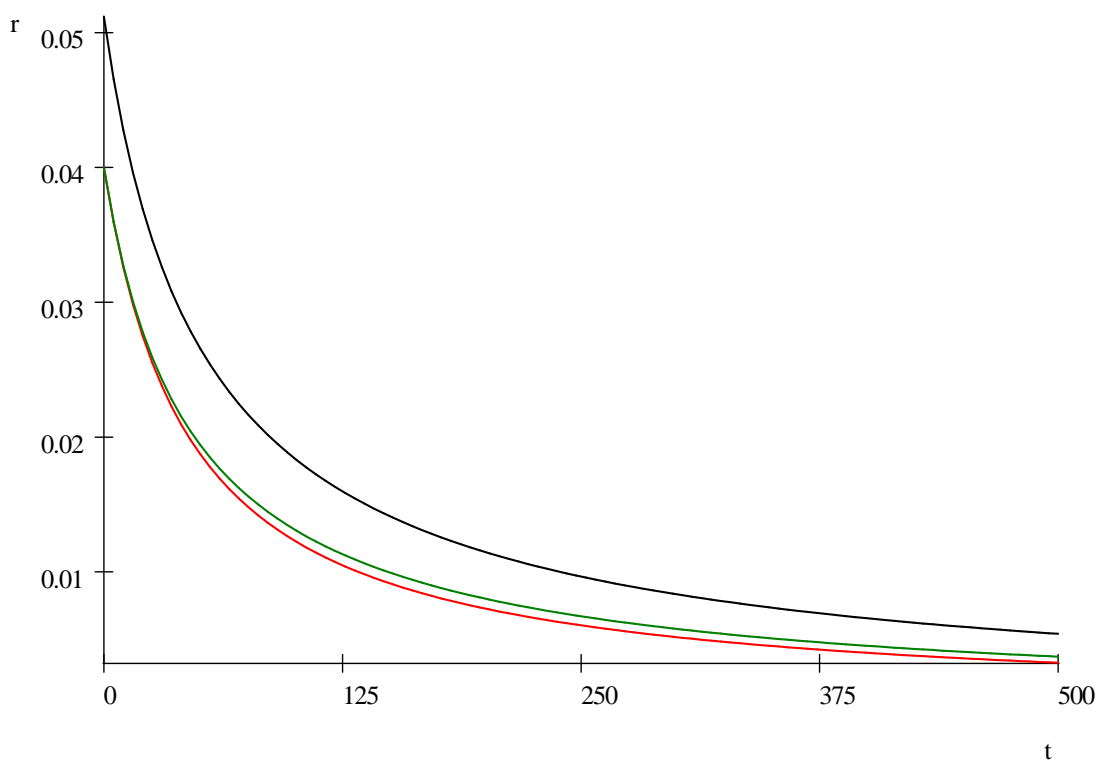

Figure 3: This figure represents the marginal discount rate curve $r_{t}=$ $\sum_{i=1}^{N} \frac{w_{i} \rho_{i} \exp \left(-r^{i} t\right)}{\sum_{j=1}^{N} w_{j} \rho_{j} \exp \left(-r^{j} t\right)} r^{i}=\frac{\alpha_{1}+1}{\beta_{1}+t}+\frac{\alpha_{2}}{\beta_{2}+t}$ obtained through our calibration (upper curve) and compares it to the discount rate curve $r_{t}=\frac{\alpha}{\beta+t}$ of Weitzman (2001) (lower curve). The intermediate curve represents, with our calibration, the unweighted average $\sum_{i=1}^{N} \frac{w_{i} \exp \left(-r^{i} t\right)}{\sum_{j=1}^{N} w_{j} \exp \left(-r^{j} t\right)} r^{i}=\frac{\alpha_{1}}{\beta_{1}+t}+\frac{\alpha_{2}}{\beta_{2}+t}$. It is clear that the difference between our discount rate curve and Weitzman (2001)'s curve mainly results from the fact that, contrarily to the certainty equivalent approach, more impatient experts are more heavily weighted in the equilibrium approach. 


\begin{tabular}{|c|c|c|c|c|c|}
\hline Time period & Name & $\begin{array}{c}\text { Numerical } \\
\text { value }\end{array}$ & $\begin{array}{c}\text { Approx. } \\
\text { rate }\end{array}$ & $\begin{array}{l}\text { Weitzman's } \\
\text { num. value }\end{array}$ & $\begin{array}{c}\text { Weitzman's } \\
\text { appr. rate }\end{array}$ \\
\hline Within years 1 & Immediate & $4.99 \%$ & $5 \%$ & $3.89 \%$ & $4 \%$ \\
\hline to 5 hence & Future & & & & \\
\hline Within years 6 & Near & $4.23 \%$ & $4 \%$ & $3.22 \%$ & $3 \%$ \\
\hline to 25 hence & Future & & & & \\
\hline Within years 26 & Medium & $2.82 \%$ & $3 \%$ & $2.00 \%$ & $2 \%$ \\
\hline to 75 hence & Future & & & & \\
\hline Within years 76 & Distant & $1.50 \%$ & $1.5 \%$ & $0.97 \%$ & $1 \%$ \\
\hline to 300 hence & Future & & & & \\
\hline Within years & Far-Distant & $0.16 \%$ & $0 \%$ & $0.08 \%$ & $0 \%$ \\
\hline more than 300 hence & Future & & & & \\
\hline
\end{tabular}

Table 1 - Approximate recommended sliding-scale discount rates

This table compares for different time periods the recommended discount rates that result from our approach and those resulting from Weitzman (2001)'s approach. These rates are computed recursively. For the first period, we compute the rate that, if applied continuously from date 0 to the middle of the period would lead to the discount rate for that maturity. For next periods, we compute the rate that, if applied continuously from the beginning of the period to the middle of the period and compounded with the rates already computed for previous periods would lead to the discount rate for that maturity. The exact as well as approximate (recommended) results are then provided for both approaches. 